\title{
Experimental validation of strong directional selectivity in nonsymmetric metallic gratings with a subwavelength slit
}

Semih Cakmakyapan, Humeyra Caglayan, Andriy E. Serebryannikov, and Ekmel Ozbay

Citation: Appl. Phys. Lett. 98, 051103 (2011);

View online: https://doi.org/10.1063/1.3552675

View Table of Contents: http://aip.scitation.org/toc/apl/98/5

Published by the American Institute of Physics

\section{Articles you may be interested in}

Cascaded metasurfaces for complete phase and polarization control

Applied Physics Letters 102, 231116 (2013); 10.1063/1.4810873

Dual-band asymmetric transmission of linear polarization in bilayered chiral metamaterial Applied Physics Letters 102, 191905 (2013); 10.1063/1.4805075

Integrating polarization conversion and nearly perfect absorption with multifunctional metasurfaces Applied Physics Letters 110, 171903 (2017); 10.1063/1.4982240

Broadband diodelike asymmetric transmission of linearly polarized light in ultrathin hybrid metamaterial Applied Physics Letters 105, 201103 (2014); 10.1063/1.4902162

High-efficiency spoof plasmon polariton coupler mediated by gradient metasurfaces

Applied Physics Letters 101, 201104 (2012); 10.1063/1.4767219

One-way total reflection with one-dimensional magneto-optical photonic crystals

Applied Physics Letters 90, 121133 (2007); 10.1063/1.2716359

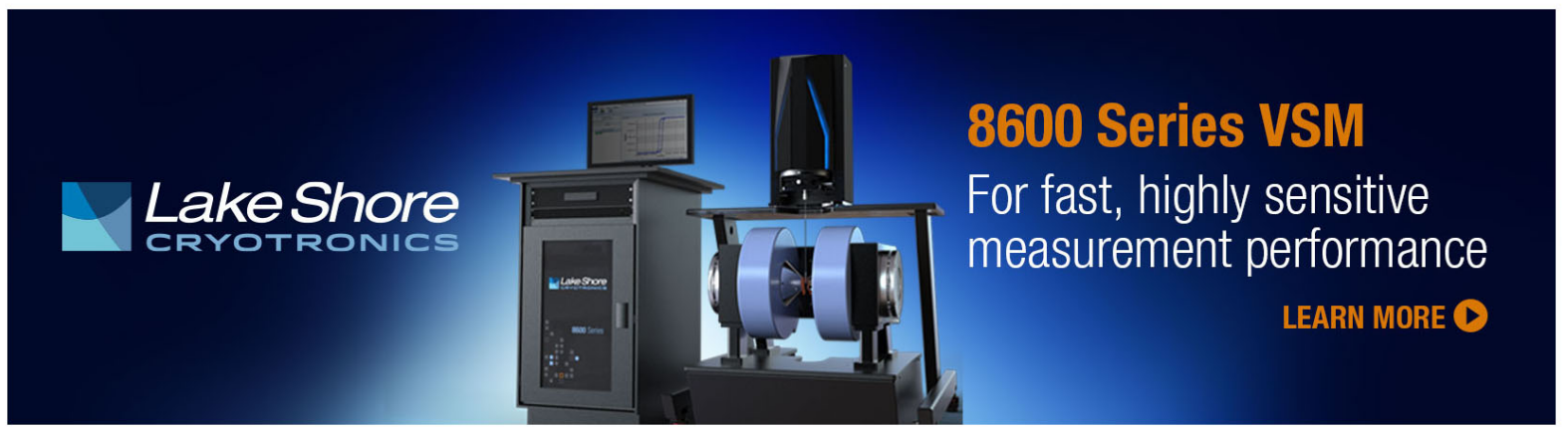




\title{
Experimental validation of strong directional selectivity in nonsymmetric metallic gratings with a subwavelength slit
}

\author{
Semih Cakmakyapan, ${ }^{1, a)}$ Humeyra Caglayan, ${ }^{2}$ Andriy E. Serebryannikov, ${ }^{3}$ and \\ Ekmel Ozbay ${ }^{1}$ \\ ${ }^{1}$ Department of Physics, Department of Electrical and Electronics Engineering, and Nanotechnology \\ Research Center, Bilkent University, Bilkent, 06800 Ankara, Turkey \\ ${ }^{2}$ Department of Electrical and Systems Engineering, University of Pennsylvania, Philadelphia, \\ Pennsylvania 19104, USA \\ ${ }^{3}$ Hamburg University of Technology, E-3, D-21071 Hamburg, Germany
}

(Received 2 September 2010; accepted 8 January 2011; published online 2 February 2011)

\begin{abstract}
Strong directional selectivity is theoretically predicted and experimentally validated at the microwave frequencies in the beaming regime for a single subwavelength slit in nonsymmetric metallic gratings with double-side corrugations. The operation regime can be realized at a fixed angle of incidence when the surface-plasmon assisted transmission is significant within a narrow range of observation angles, if illuminating one of the grating interfaces, and tends to vanish for all observation angles, if illuminating the opposite interface. The studied effect is connected with asymmetry (nonreciprocity) in the beaming that occurs if the surface plasmon properties are substantially different for the two interfaces being well isolated from each other. () 2011 American Institute of Physics. [doi:10.1063/1.3552675]
\end{abstract}

The electromagnetic response of subwavelength apertures has been a subject of extensive study since that time when Bethe showed that the diffraction patterns for smooth perfect conducting metallic screens with subwavelength holes are isotropic in the special case of unpolarized incident light. ${ }^{1}$ On the other hand, optical properties of metallic surfaces can be explained in terms of surface plasmons, which represent, in fact, collective longitudinal electron oscillations. Since the wave vector of the incident light is smaller than that of a surface plasmon mode, light incident from free space cannot couple to the surface plasmon modes at smooth metallic surfaces. ${ }^{2}$ Surface-plasmon originated extraordinary transmission has been in focus of interest starting from the pioneering work of Ebbesen et al. ${ }^{3}$ In particular, it was theoretically demonstrated that surface plasmon like modes are achievable even in the perfect conductor limit whatever are the size and frequency range, if the surface is patterned with subwavelength holes. ${ }^{4}$ Hibbins et al. experimentally studied such modes in near-perfect conductors with holes in the microwave regime. ${ }^{5}$

It was shown that the transmitted beam can be enhanced by means of periodic corrugations placed at one or both interfaces. ${ }^{6}$ Introducing a grating at the illumination (input) interface provides the coupling of a free-space electromagnetic wave to the surface plasmon modes, due to which transmission through the subwavelength apertures can be enhanced. ${ }^{6-8}$ As well as the transmission enhancement, beaming and localization of transmitted beam to a spatial region can be obtained owing to placing a properly designed grating at the output interface. ${ }^{7,9-11}$ Furthermore, it was shown that steering and tuning the direction of the transmitted beam are possible by placing corrugations at the output interface asymmetrically with respect to the slit axis. ${ }^{12-14}$ Strong asymmetry (nonreciprocity) in reflection has been considered in detail for nonsymmetric structures that repre-

${ }^{\text {a)} E l e c t r o n i c ~ m a i l: ~ s e m i h c @ b i l k e n t . e d u . t r . ~}$ sent a subwavelength hole array in a metallic film deposited on a dielectric substrate. ${ }^{15,16}$ It appears due to changing illumination side, while the conditions of excitation of surface plasmons at two interfaces are different.

Recently, surface-plasmon originated unidirectional transmission has been theoretically studied for the gratings with a single slit, which are nonsymmetric due to that they have double-side corrugations with different periods at the front and back sides. ${ }^{17}$ In fact, this mechanism exploits the effect of isolation, which manifests itself in such structures in that the transmission process can be separated into three relatively independent steps, i.e., coupling in, transmission through the hole, and coupling out. ${ }^{18}$ In particular, this means that since the surface plasmon appearance depends on corrugation parameters, the exit interface is mainly responsible for the beaming into the exit half-space, while the input interface might just slightly affect the field topology. This may lead to strong asymmetry in diffraction patterns and angledependent transmission spectra for two opposite illumination directions. In turn, the surface plasmons excited at the input interface are expected to be mainly responsible for the strength of extraordinary transmission, so that this effect can also be asymmetric. At the same time, the transmission mechanism studied in Ref. 17 can be interpreted as an extension of that suggested earlier for the gratings based on the two-dimensional photonic crystals ${ }^{19}$ and gratings containing solid metallic or wire slabs with the effective index of refraction $0<n<1 .{ }^{20}$ However, for the mechanism in Ref. 17, surface plasmons are necessary, rather than specific dispersion features of the waves propagating in the structure/ material, on which the unidirectional gratings in Refs. 19 and 20 are based.

In this letter, we theoretically and experimentally study directional selectivity, which appears at microwave frequencies in transmission through the subwavelength slit in the metallic slab with the corrugated interfaces at fixed angle of incidence, $\theta$. Based on the comparison of several theoretical 


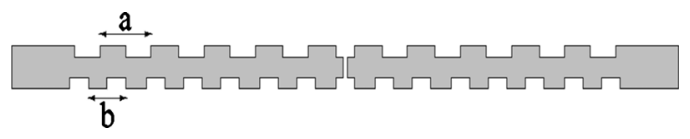

FIG. 1. Schematic of the metallic (aluminum) gratings. Slit width is $2 \mathrm{~mm}$, slit length between the interfaces is $8 \mathrm{~mm}$, and groove depth is $4 \mathrm{~mm}$. Grating periods of the back and front sides are denoted by $a$ and $b$, respectively.

grating performances, the role of the grating nonsymmetry in achieving unidirectional transmission is demonstrated, and the performances, which are particularly appropriate for the purposes of experimental investigation, are selected. We restrict our consideration to the gratings with double-side corrugations, where surface plasmons might appear on the both sides.

Figure 1 shows the geometry of gratings, which are assumed to be made of aluminum (Al), with a subwavelength slit at the center. Three samples were chosen to be in focus of our study, based on the comparison of several similar structures. Further, we refer to them as samples A-C. Grating periods of the front-side and back-side interfaces are different for the all three samples. However, they are kept symmetric with respect to the slit axis. For sample A, we assume that $a=26 \mathrm{~mm}$ and $b=16 \mathrm{~mm}$. For sample B, $a=22 \mathrm{~mm}$ and $b=16 \mathrm{~mm}$. Finally, for sample C, we set $a=22 \mathrm{~mm}$ and $b=14 \mathrm{~mm}$. The structures are illuminated with a TMpolarized plane wave (magnetic field vector is parallel to the slit).

Before performing microwave experiment, we compare simulation results obtained by using finite-difference time domain technique for samples A-C. The transmission calculated $50 \mathrm{~cm}$ away from the center of the slit is presented in Fig. 2, for the observation angle $\Phi=0$ that is associated with the direction which is perpendicular to the grating. The incident Gaussian beam has been assumed to be $9 \mathrm{~cm}$ wide. The value of $\theta$ was chosen so that the angle-dependent resonance frequencies differ substantially, depending on which side is illuminated. The chosen value of $\Phi$ (nearly) corresponds to the angle where strong beaming can appear. For sample A, the strongest transmission is observed at $f=9.8 \mathrm{GHz}$ in the case of the front-side illumination and at $f=15.7 \mathrm{GHz}$ in the case of the back-side illumination. In turn, transmission is substantially weaker at $f=9.8 \mathrm{GHz}$ in the case of the back-

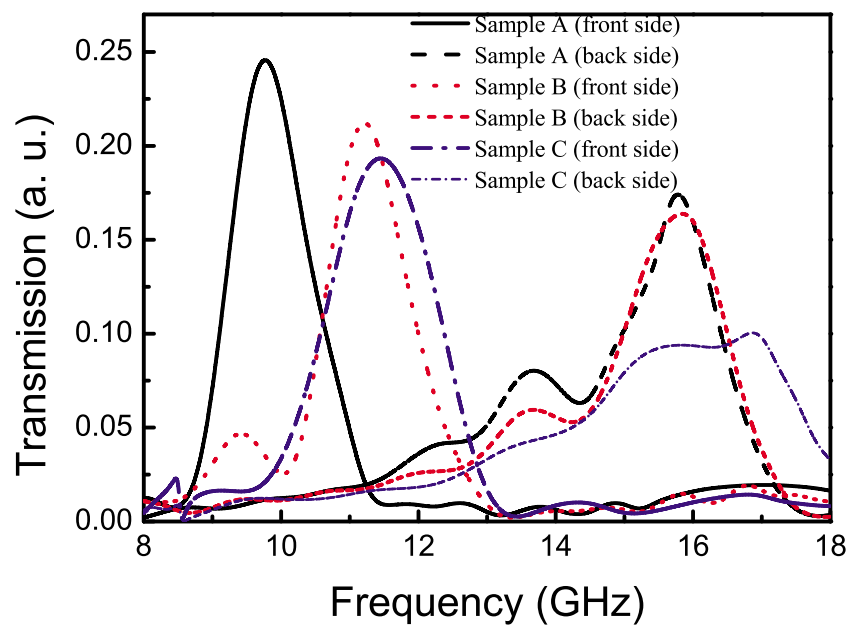

FIG. 2. (Color online) Calculated transmission for samples A-C at $\theta=30^{\circ}$. Illumination side is specified in brackets.

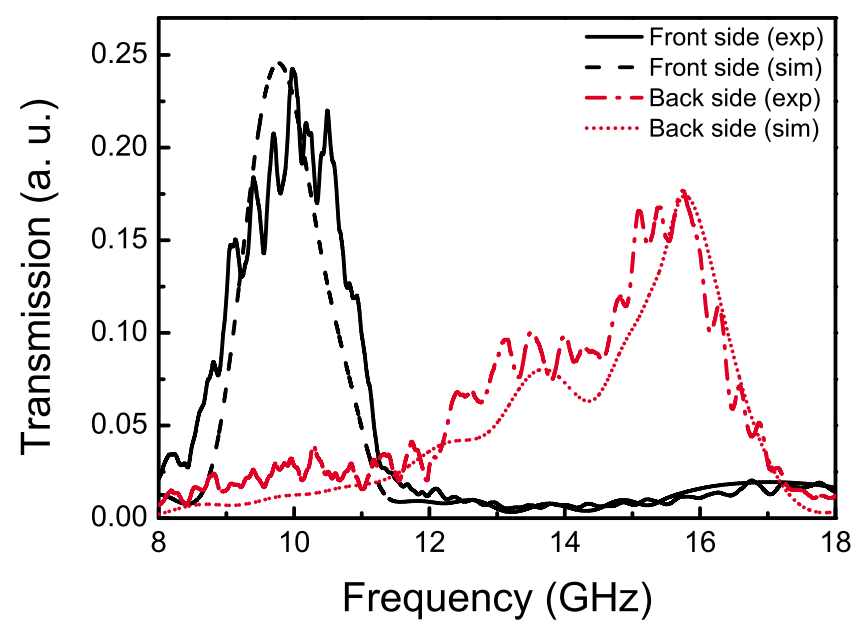

FIG. 3. (Color online) Experimental and simulated transmission results for sample A at $\theta=30^{\circ}$.

side illumination and near $f=15.7 \mathrm{GHz}$ in the case of the front-side illumination. In the last two cases, transmission is weak for all $\Phi$. Hence, strong directional selectivity occurs in the vicinity of these two peculiar frequencies. Similar features are observed for samples B and C. The maximal transmittance is obtained for these samples at the front-side illumination in the vicinity of $f=11 \mathrm{GHz}$, while they show a smaller period $a$ than sample A. The maxima locations and transmittance values observed in this case can be considered as confirmation of the dominant role of the exit interface for the beaming. The same remains true concerning those for samples A and B in the vicinity of $f=15.7 \mathrm{GHz}$, at the backside illumination. From the obtained results, the role of nonsymmetry of the grating $(a \neq b)$ as a necessary condition of unidirectional transmission is clearly seen.

Figure 3 compares the results of experiments and simulations for sample A at $\Phi=0$. The experimental results are obtained by sending an incident beam, whose source (a horn antenna) is $20 \mathrm{~cm}$ away from the input interface, and measuring transmission $50 \mathrm{~cm}$ away from the output interface. Measurements were carried out in the frequency range from 8 to $18 \mathrm{GHz}$ by using two standard horn antennas and an HP $8510 \mathrm{C}$ network analyzer. The main attention has been paid to the detection of cases when transmission strongly depends on the illumination direction at fixed $\theta$. In Fig. 3, good coincidence between the simulation and experimental results is observed.

In order to validate that unidirectional transmission appears in the beaming regime, we investigated details of the angular dependence of transmission near the frequencies, at which the maxima are observed in Figs. 2 and 3. In Fig. 4, the calculated and measured $\Phi$-dependences of transmission are presented for sample A. The coincidence is good, while the unidirectional transmission is strongly pronounced. The half-power bandwidth in the angle domain is nearly equal to $9^{\circ}$ at the front-side illumination.

Finally, the experimental and simulation results are presented in Fig. 5 for sample B at $f=11.2 \mathrm{GHz}$ and $\theta=0$. Again, the coincidence is quite good. For the front-side illumination, the beaming is observed with the transmission maximum at $\Phi=0$. However, when the structure is illuminated from the back side, no beaming is observed while transmission remains significant. Despite this, rather strong 


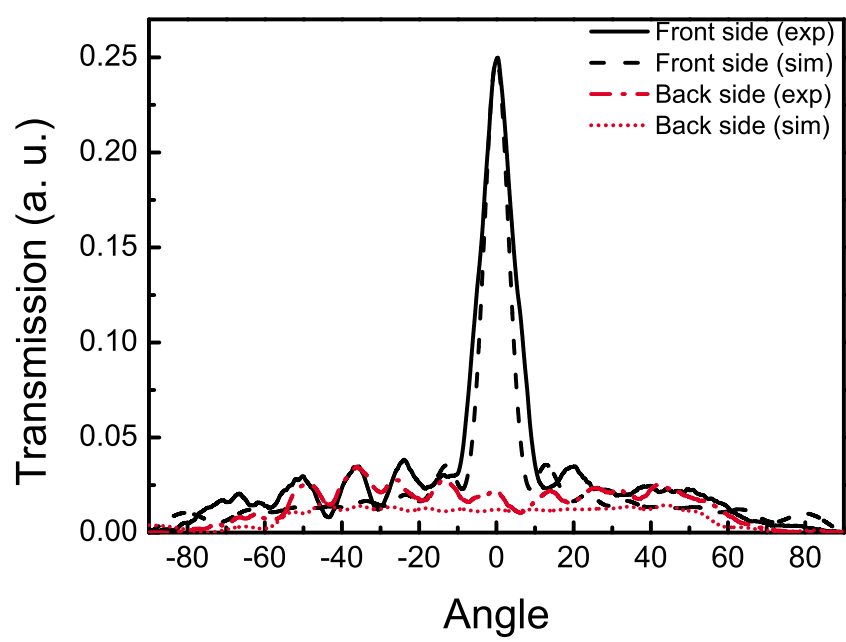

FIG. 4. (Color online) Experimental and simulated angular distributions of transmission at $f=9.8 \mathrm{GHz}$ for sample A at $\theta=30^{\circ}$. Positive angles represent the right-hand side of the structure, while negative angles represent the left-hand side of the structure.

directional selectivity occurs within a limited range of observation angles. At $20^{\circ}<|\Phi|<30^{\circ}$, transmission is significant at the back-side illumination but relatively weak at the frontside illumination. At the same time, there is no feature of one-way transmission in the vicinity of $\Phi=0$.

The obtained results promise that a diodelike device can be designed after a proper optimization, opening a route to a new class of unidirectional devices which operate in the beaming regime. It is noteworthy that more various regimes of strong unidirectional transmission can be obtained in the gratings based on the advanced structures like three-

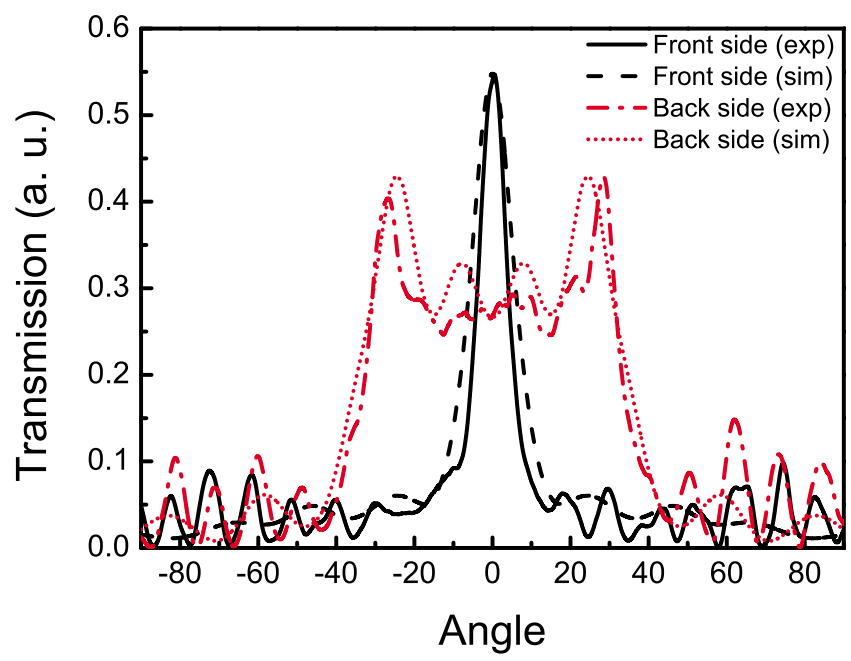

FIG. 5. (Color online) Experimental and simulated angular distributions of transmission at $f=11.2 \mathrm{GHz}$ for sample B at $\theta=0$. dimensional photonic crystals ${ }^{21}$ and photonic crystals with defects. $^{22}$ In turn, advanced performances of the studied structures are possible, based on the analogy with the mentioned photonic crystals. A smaller structure thickness $D$ than in Refs. 19 and 20 is sufficient in our gratings for obtaining a well pronounced unidirectional transmission at fixed $\theta$. For samples A-C, $D=16 \mathrm{~mm}$, and $D / \lambda=0.52$, where the freespace wavelength $\lambda$ corresponds to $f=9.8 \mathrm{GHz}$.

To summarize, we validated the unidirectional beaming that appears at a single subwavelength slit in nonsymmetric metallic gratings at microwave frequencies. The role of nonsymmetry of the grating and, hence, of the surface plasmons at the input and exit interfaces in the appearance of the strong directional selectivity was studied theoretically and experimentally. The obtained results demonstrated how the exit interface affects transmission spectra and spatial features of the outgoing waves.

This work was supported by the European Union under the projects PHOME, ECONAM, N4E, and TUBITAK under Project Nos. 109E301, 107A004, and 107A012 and by DPT under the project DPT-HAMIT. E.O. also acknowledges partial support from the Turkish Academy of Sciences. A.S. thanks the DFG for support under Project No. SE1409/2-1.

${ }^{1}$ H. A. Bethe, Phys. Rev. 66, 163 (1944).

${ }^{2} \mathrm{H}$. Raether, Surface Plasmons on Smooth and Rough Surfaces and on Gratings (Springer, Berlin, 1988).

${ }^{3}$ T. W. Ebbesen, H. J. Lezec, H. F. Ghaemi, T. Thio, and P. A. Wolff, Nature (London) 391, 667 (1998).

${ }^{4}$ J. Pendry, L. Martin-Moreno, and F. Garcia-Vidal, Science 305, 847 (2004).

${ }^{5}$ A. Hibbins, B. Evans, and J. Sambles, Science 308, 670 (2005).

${ }^{6}$ T. Thio, K. Pellerin, R. Linke, H. Lezec, and T. Ebbesen, Opt. Lett. 26, 1972 (2001).

${ }^{7}$ H. J. Lezec, A. Degiron, E. Devaux, R. A. Linke, L. Martin-Moreno, F. J. Garcia-Vidal, and T. W. Ebbesen, Science 297, 820 (2002).

${ }^{8}$ H. Caglayan, I. Bulu, and E. Ozbay, Opt. Express 13, 1666 (2005).

${ }^{9}$ E. Ozbay, Science 311, 189 (2006).

${ }^{10}$ H. Caglayan, I. Bulu, and E. Ozbay, J. Opt. Soc. Am. B 23, 419 (2006).

${ }^{11}$ Z. Li, K. Aydin, and E. Ozbay, Appl. Phys. Lett. 91, 121105 (2007).

${ }^{12}$ S. Kim, H. Kim, Y. Lim, and B. Lee, Appl. Phys. Lett. 90, 051113 (2007).

${ }^{13}$ D. Lin, T. Cheng, C. Chang, J. Yeh, J. Liu, C. Yeh, and C. Lee, Opt. Express 15, 2585 (2007).

${ }^{14}$ H. Caglayan, I. Bulu, and E. Ozbay, J. Appl. Phys. 104, 073108 (2008).

${ }^{15}$ E. Altewischer, M. P. van Exter, and J. P. Woerdman, Opt. Lett. 28, 1906 (2003).

${ }^{16}$ M. Sarrazin and J. P. Vigneron, Phys. Rev. B 70, 193409 (2004).

${ }^{17}$ S. Cakmakyapan, A. E. Serebryannikov, H. Caglayan, and E. Ozbay, Opt. Lett. 35, 2597 (2010)

${ }^{18}$ A. Degiron and T. W. Ebbesen, Opt. Express 12, 3694 (2004).

${ }^{19}$ A. E. Serebryannikov, Phys. Rev. B 80, 155117 (2009).

${ }^{20}$ A. E. Serebryannikov and E. Ozbay, Opt. Express 17, 13335 (2009).

${ }^{21}$ E. Özbay, G. Tuttle, R. Biswas, K. M. Ho, J. Bostak, and D. M. Bloom, Appl. Phys. Lett. 65, 1617 (1994).

${ }^{22}$ E. Özbay, G. Tuttle, J. S. McCalmont, R. Biswas, M. Sigalas, C. M. Soukoulis, and K. M. Ho, Appl. Phys. Lett. 67, 1969 (1995). 